\title{
Hydatid cyst of the uterus
}

\author{
A. Başgül11, Z. N. Kavak², H. Gökaslan² and S. Küllü̈ ${ }^{3}$ \\ ${ }^{1}$ Clinic of Obstetrics and Gynecology, Batman Social Security Hospital, Batman \\ ${ }^{2}$ Department of Obstetrics and Gynecology, and \\ ${ }^{3}$ Department of Pathology, School of Medicine of Marmara University, Istanbul, Turkey
}

\begin{abstract}
Background: Hydatidosis is a common zoonosis that affects a large number of humans and animals, especially in poorly developed countries. The infesting parasite has four forms named Echinococcus granulosis, E. multilocularis, $E$. vogeli and $E$. oligarthrus (very rare in humans). The most frequently involved organs are liver followed by the lung. The involvement of the genital tract is rare and the occurrence in the uterus is an extreme rarity. We report a case of hydatid cyst in the uterus.
\end{abstract}

Case: A 70-year-old female with a history of hydatid cysts of the liver, was admitted to hospital after complaining of low abdominal pains. On physical and gynecological examinations, no pathological finding was detected. However, the uterus was significantly large for a postmenopausal patient. Transvaginal sonography (TS) revealed a cystic mass in the uterus with a size of $7 \times 6 \mathrm{~cm}$. After further examinations a subtotal hysterectomy was performed. Microscopic examination showed scolices of Echinococcus granulosis.

Conclusion: Hydatid cysts in the genital tract are rare and the occurrence in the uterus is an extreme rarity. Differentiation between hydatid cyst and malignant disease of the related organ is difficult. To avoid misdiagnosis, a careful examination of pelvic masses should be carried out in endemic areas for detection of hydatid cysts.

Key words: Hydatid Cyst; Uterus; ECHINOCOCCUS GRANULOSIS

Hydatidosis is a common zoonosis, which may be endemic in some parts of the world ${ }^{1}$. The disease, especially in poorly developed countries, affects large number of humans and animals. The infesting parasite is the smallest taenia adult of the cestodes which has the largest larva. It has four forms named Echinococcus granulosis, E. multilocularis, E. vogeli and E. oligarthrus (very rare in humans). The definitive hosts are canids, mostly dogs. The tapeworms grow into adulthood in the host intestines and release their eggs in the feces. These eggs are ingested by intermediate hosts (sheep, cattle, pigs, horses, camels and humans). The larvae migrate through the intestinal wall invading other tissues. The most frequently involved organ is liver (75\%), followed by the lung (15\%) and the remainder of the body $(10 \%)^{1,2}$. The involvement of the genital tract is rare and the occurrence in the uterus is an extreme rarity. Clinical history, serologic tests and various imaging techniques such as ultrasonography (USG), computed tomography (CT) and magnetic resonance imaging (MRI) can help make the diagnosis. It may be confused with malignancies of the affected organs. The main treatment is

Correspondence to: Zehra N. Kavak, MD, Department of Obstetrics and Gynecology, School of Medicine of Marmara University, Altunizade-81190, Uskudar, Istanbul, Turkey 
surgical excision followed by mebendazole for four months.

\section{CASE}

A 70-year-old postmenopausal woman, gravida 5, para 4, was admitted to Batman Social Security Hospital in the southeastern part of Anatolia, with low abdominal pain lasting for two days. Two successive surgeries had already been performed on the patient for hydatid cyst of the liver in 1997 and 1999. The patient had a history of hypertension and diabetes mellitus. On physical examination, no pathological finding was noted except previous surgical scars on the abdomen. The uterus was significantly large for a postmenopausal patient. No adnexal mass was palpated during the pelvic exam. The pulse was normal and blood pressure was slightly elevated. Routine emergency laboratory findings such as hemogram, hematocrit and urinalysis were also found insignificant for diagnosis.

Following the physical and gynecologic examination, the patient had pelvic USG and CT scans. The USG revealed a cystic area with regular borders in the uterus with a size of $7 \times 6 \mathrm{~cm}$. A subsequently-performed CT scan disclosed the radiolucent mass with well-circumscribed borders in the cavity of the uterus. The size of the lesion, resembling a fluid-filled cavity with homogenous appearance, was measured as $82.2 \times 88.4 \mathrm{~mm}$ in pelvic transverse section. There was an involvement of the myometrium resulting in an obvious thinning of the wall of the uterus. Primarily, the diagnosis of uterine malignancy should be considered in a postmenopausal patient with these findings until proven otherwise. The spaces between the bladder and rectum were clear as well as parametria bilaterally. No extension to the cervix was noted (Figure 1).

The patient underwent an exploratory laparatomy for the diagnosis of the unusual mass within the body of the uterus. An endometrial biopsy or diagnostic curettage to rule out malignancy was not performed since the mass in the cavity was cystic and the previous history of hydatid cysts had raised the suspicion of this diagnosis. A subtotal hysterectomy was performed due to technical difficulties during surgery and inconvenient medical conditions. Subsequently, the patient was referred to the Obstetrics and Gynecology Clinic of University of Marmara for further postoperative care. The postoperative course was uneventful and the patient was discharged in perfect health.

The specimen was evaluated in the pathology department of the University of Marmara and reported as hydatid cyst of the uterus. The subtotal hysterectomy specimen measured $8 \times 8 \times 7.5 \mathrm{~cm}$ with a smooth peritoneal surface. Cut section revealed a unilocular white cyst with a diameter of

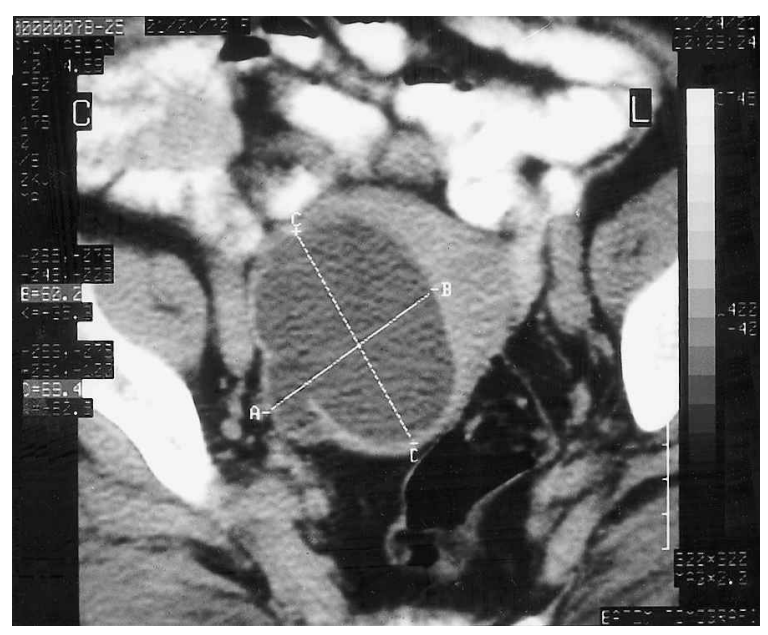

Figure I CT image showing a cystic mass within the uterine cavity

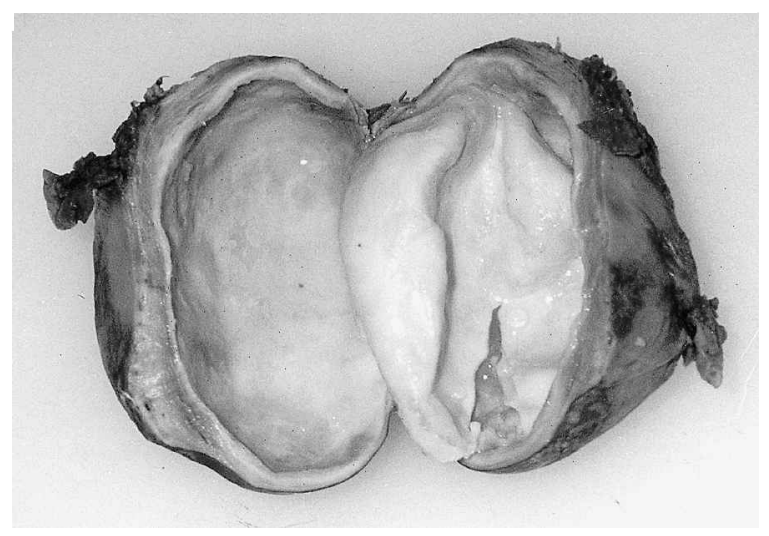

Figure 2 A cut in surface of the uterine cavity shows white cyst with surrounding fibrous capsule 
$7.5 \mathrm{~cm}$ and opalescent fluid within. This resulted in a very thin-walled myometrium measuring $0.1 \mathrm{~cm}$ in thickness in some areas (Figure 2). Microscopic examination showed scolices of Echinococcus granulosis with adjacent laminated membrane in the inner surface of the thinwalled myometrium (Figure 3). Currently, the patient is under follow-up at Marmara University for recurrence.

\section{CONCLUSION}

The unusual localization of hydatid cyst in the brain, heart, pericardium, kidney, intraperitoneum, retroperitoneum, bone, soft tissue and breast as rare sites has been discussed in the literature ${ }^{2}$.

The localization of the hydatid cyst in the uterus is an extremely rarely encountered entity and highly interesting. Gueddana and colleagues ${ }^{3}$ reported a case with intrauterine hydatidosis whose hydatid vesicles were found in the vagina and a total hysterectomy was carried out. Okumus and co-workers ${ }^{4}$ also reported a case in which the primary involvement was uterus and the diagnosis was confirmed by microscopic studies after the surgery.

The correct diagnosis of hydatidosis is very difficult because of the striking resemblance between the hydatid cyst and malignant disease of the related organ. Unfortunately, it was difficult to make the correct diagnosis in our case preoperatively despite the patient having given the history of hydatid cysts. If the physician lacks the high index of suspicion, the lesion may be misdiagnosed as a pelvic malignancy. Therefore, hydatid cysts should be considered in the differential diagnosis of cystic pelvic masses, especially in areas where the disease is endemic.
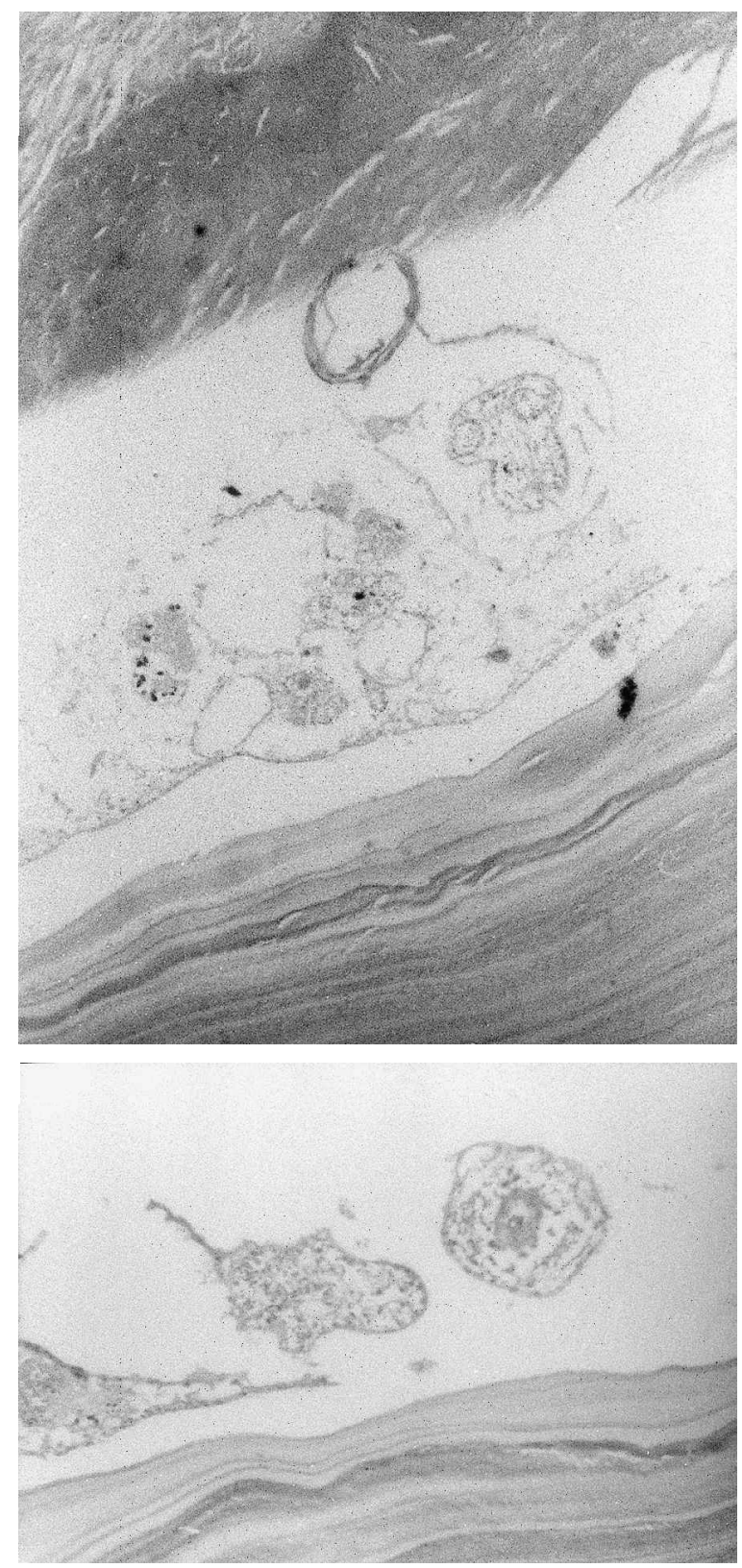

Figure 3 Microphotograph of the lesion reveals laminated membrane and the scolices surrounded by fibrous capsule and atrophic myometrium. 


\section{REFERENCES}

1. Bouree P. Hydatidosis: dynamics of transmission. World J Surg 2001;25:4-9

2. Engin G, Acunas B, Rozanes I, et al. Hydatid disease with unusual localization. Eur Radiol 2000;10: 1904-12

ReCEIVED 05/14/01; ACCEPTED 01/07/02
3. Gueddana F, Chemmen-Lebbene L, Lebbi I, et al. Intrauterine hydatidosis. A case report. J Gynecol Obstet Biol Reprod (Paris) 1990;19:725-7

4. Okumus Y, Tayyar M, Patiroglu T, et al. Uterine hydatid cyst. Int J Gynaecol Obstet 1994;45:51-3 


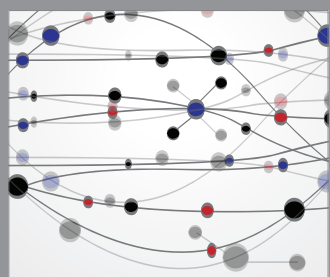

The Scientific World Journal
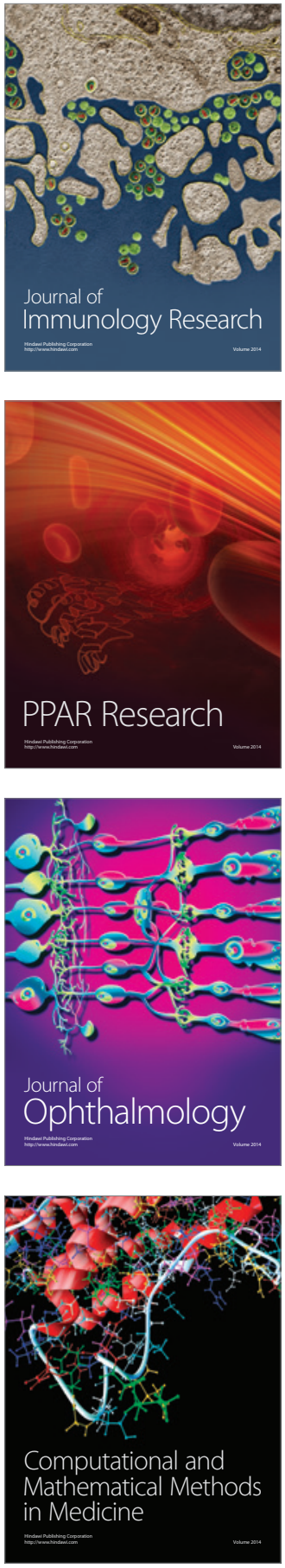

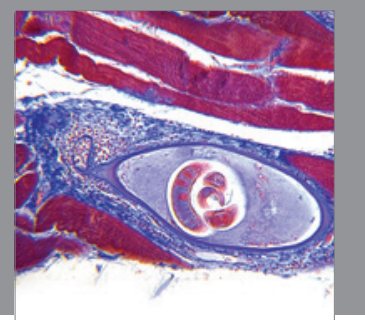

Gastroenterology

Research and Practice
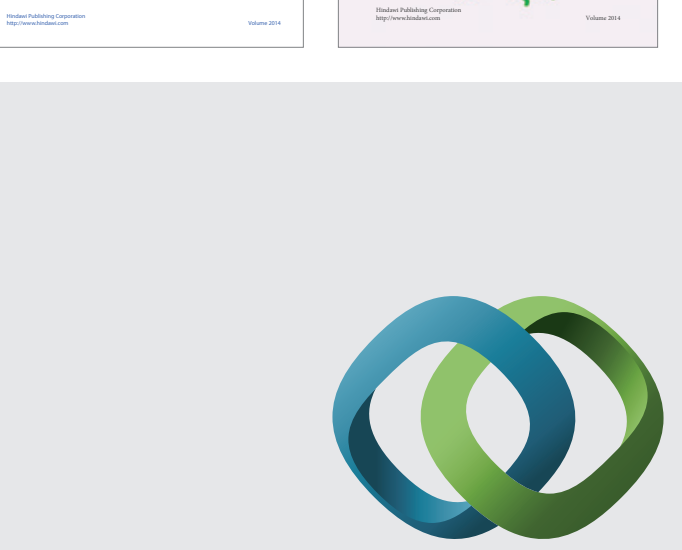

\section{Hindawi}

Submit your manuscripts at

http://www.hindawi.com
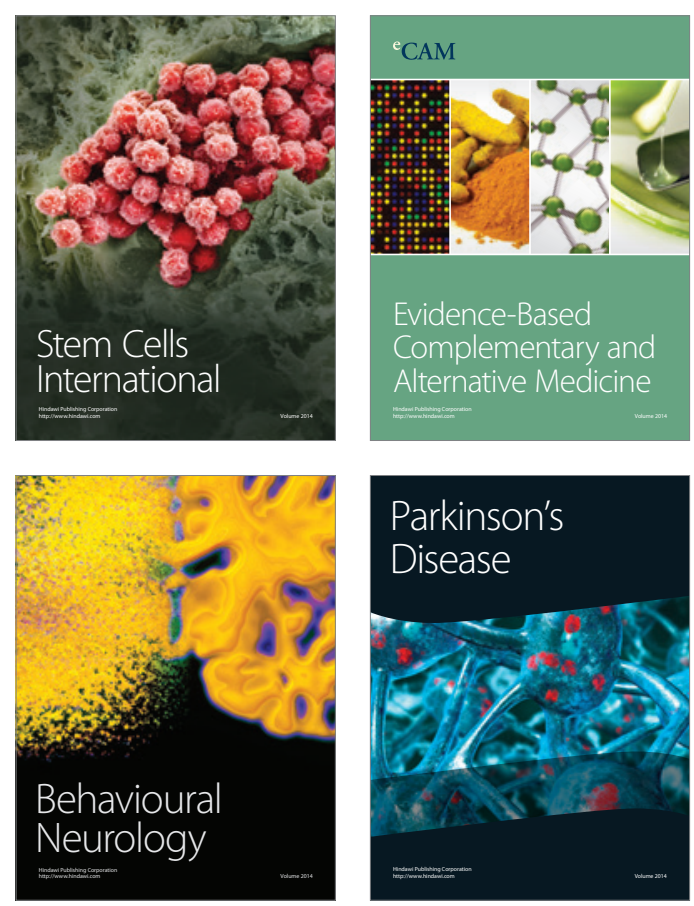

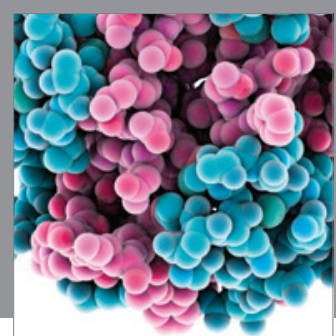

Journal of
Diabetes Research

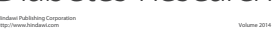

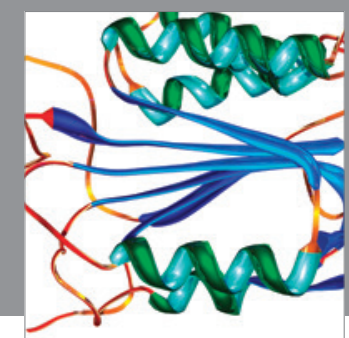

Disease Markers
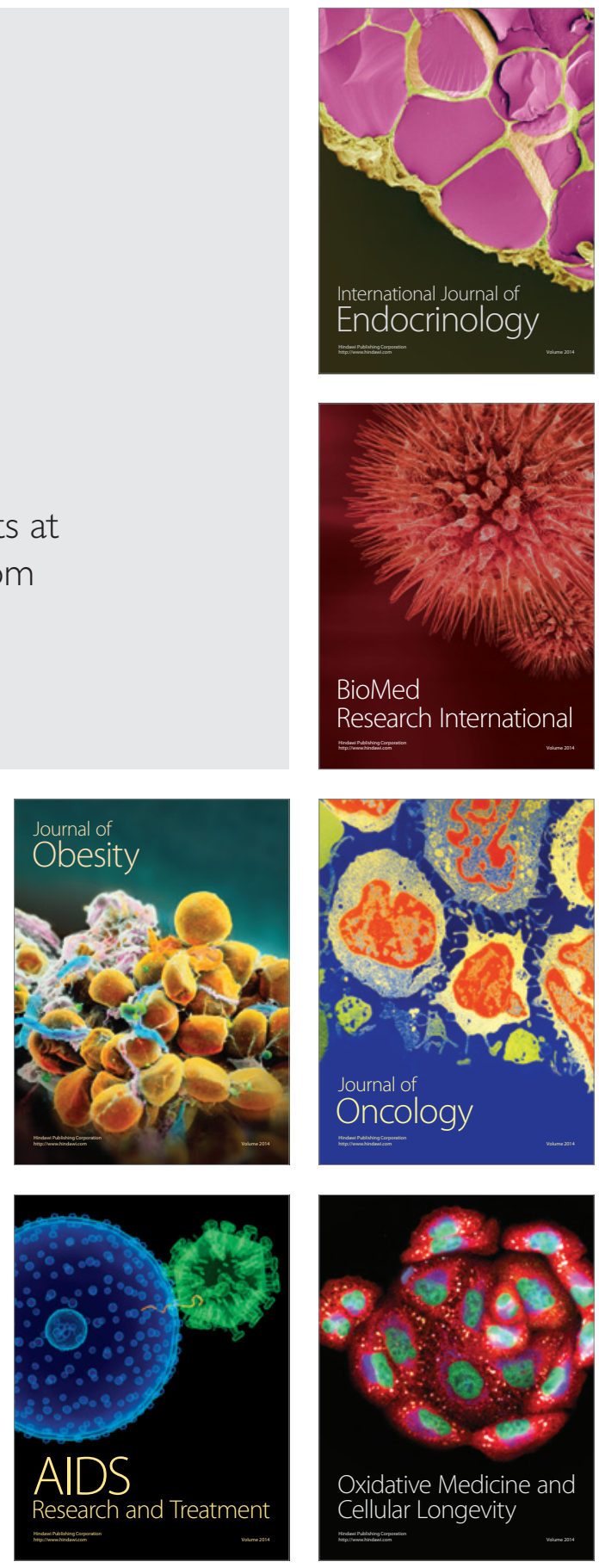